ARTÍCULO DE INVESTIGACIÓN

\title{
Análisis del crecimiento e incremento de cinco pináceas de los bosques de Durango, México
}

\author{
Sacramento Corral R. \\ José de Jesús Návar Cháidez.
}

\section{RESUMEN}

\begin{abstract}
Diversas ecuaciones de crecimiento e incremento en diámetro, altura y volumen fueron ajustadas y validadas para las especies Pinus durangensis, P. cooperi, P. leiophylla, $P$. engelmannii y $P$. herrerae que se distribuyen naturalmente en la región de El Salto, Durango, México. Los resultados mostraron que a través del modelo de Chapman-Richards la primera especie presenta los crecimientos e incrementos mayores cuando el crecimiento se estima a nivel grupo de árboles e individualmente. El trabajo enfatiza la necesidad de establecer parcelas permanentes de muestreo para calibrar estas tecnologías y ser usadas en modelos de crecimiento al nivel de rodales completos, grupo de árboles o árboles individuales.
\end{abstract}

PALABRAS CLAVE:

Árboles individuales, Durango, funciones de crecimiento e incremento, grupo de árboles, México, pináceas, Pinus.

\begin{abstract}
Several growth and increment in diameter, height and volume equations were fitted and validated for the pine species Pinus cooperi, $P$. durangensis, $P$. engelmannii, $P$. leiophylla and $P$. herrerae which naturally distribute in the region of El Salto, Durango, Mexico. The results showed that by using the Chapman-Richards equation the first pine species has the highest growth and increment estimated by two different approaches. This paper points out the need to establish permanent sampling plots to calibrate these technologies to be used in growth and yield modeling at the whole stand, size class or individual tree models.
\end{abstract}

KEYWORDS:

Individual trees, Durango, growth and increment functions, group of trees, Mexico, pinaceae, Pinus. 


\section{INTRODUCCIÓN}

El manejo forestal presente requiere de estimaciones objetivas del crecimiento e incremento de los árboles del bosque. Esta información es clave en la planeación de la cosecha sustentable y en la implementación de las mejores alternativas silvícolas. El crecimiento del bosque puede ser entendido como un proceso dinámico y desde el punto de vista de un balance de masas incluye una entrada (incorporación), un movimiento (crecimiento) y una salida (mortalidad y cosecha). El crecimiento de los árboles individuales se considera como el cambio gradual en dimensiones a través del tiempo y se encuentra afectado por diversos componentes, entre los que destacan el origen de las especies (genotipo) y su entorno ambiental (Bahamóndez, 1995).

El crecimiento del bosque es difícil de pronosticar a largo plazo, pues depende de aspectos fisiológicos (tasa fotosintética y de respiración), climáticos (disponibilidad de luz, temperatura, precipitación, etc.), físicos (niveles de nutrientes en el suelo y concentraciones de bióxido de carbono en la atmósfera) y biológicos (incorporación, competencia, mortalidad, etc.) (Vanclay 1994, 1995). El crecimiento en diámetro y altura es, como consecuencia, un tanto errático anualmente aunque la tendencia sigmoidal es clara.

Clutter et al. (1983) y Vanclay (1994) mencionan que el crecimiento del bosque se puede definir objetivamente a diferentes niveles: rodal, grupo de árboles y de árboles individuales, además que puede ser definido espacial o no espacialmente. La mejor opción es aquella que resulte útil para los objetivos para los cuales los modelos son diseñados y evaluados. Es decir, un modelo puede responder con diferentes resultados a ciertas necesidades. Buckman y Shifley (1983) mencionan que el mejor modelo de crecimiento es aquel de fácil uso, preciso en el rango de datos usados para su construcción y que produce resultados biológicos realistas.
En general existen dos procedimientos para modelar el crecimiento e incremento del bosque. Uno es cuando el potencial de crecimiento es directamente expresado por las características de los árboles y del rodal, incluyendo el nivel de competencia (Martin y Ek, 1984; Wykoff et al., 1982). El otro es cuando el potencial de crecimiento está representado por árboles que crecen libres de competencia y posteriormente se ajusta un factor que modifique el potencial de crecimiento por efecto de la competencia (Shifley y Brand, 1984; Arney, 1985; Smith et al., 1992).

El modelaje del crecimiento del bosque que proporciona resultados a un nivel más detallado requiere de datos obtenidos en parcelas permanentes de muestreo, pues permiten la representación de la realidad en las diferentes etapas de desarrollo de las masas forestales. Estos modelos pueden incluir la definición matemática de la regeneración, competencia, reclutamiento y mortalidad que puede ser al nivel espacial de la masa forestal. Dado la escasez de información proveniente de parcelas permanentes de muestreo en los bosques de México, son pocos los trabajos que analizan el crecimiento con está fuente de datos (Zepeda y Domínguez, 1998). Otra aproximación es modelar el crecimiento e incremento con datos obtenidos de análisis troncales de los árboles que crecen libres de competencia. Chojnacky (1997) desarrolló un modelo de crecimiento en diámetro para árboles individuales de dos especies de Pinus y tres de Juniperus con datos de análisis troncales. Hokka y Groot (1999) derivaron un modelo de crecimiento para árboles individuales usando datos de análisis troncales de Picea mariana (Mill). Contreras et al. (1996) analizaron el crecimiento de una plantación de Rauli [Nothofagus procera] usando la técnica de análisis troncales.

Para los bosques irregulares y mixtos de Durango los estudios publicados en la literatura científica hacen referencia al modelaje del crecimiento e incremento de pocas especies usando análisis troncales. Los trabajos reportados se basan en rodales regulares monoespecíficos (Aguirre, 1987; 
Návar et al., 1996; Zepeda y Domínguez, 1998) y existe la necesidad incorporar los niveles de crecimiento e incremento que presentan las especies a nivel de conjunto de arboles o individualmente.

Los objetivos de este trabajo fueron: 1) comparar y validar ecuaciones de crecimiento e incremento a nivel grupo de árboles y 2) determinar las diferencias en crecimiento e incremento en diámetro, altura y volumen de cinco especies comerciales de pino del estado de Durango, México.

\section{MATERIALES Y MÉTODOS}

\section{Descripción general del área de estudio.}

El estudio se realizó en la región del Salto, Durango, la cual comprende los predios de la Unidad de Conservación y Desarrollo Forestal (UCODEFO) No. 6, localizándose en el sistema montañoso denominado Sierra Madre Occidental. Esta pertenece a las subprovincias Gran Meseta, cañones duranguenses y mesetas y cañones del sur. Se encuentra entre las coordenadas $23^{\circ} 30^{\prime}$ a $24^{\circ} 15^{\prime}$ de latitud Norte y $105^{\circ} 15^{\prime}$ a $105^{\circ} 45^{\prime}$ de longitud Oeste, a $100 \mathrm{~km}$ al sudoeste de la ciudad de Durango. La altura sobre el nivel del mar fluctúa entre $1400 \mathrm{~m}$ y 3 $000 \mathrm{~m}$. La región se encuentra dentro del grupo de climas templados $C$, sub-grupo de climas semicálidos $(A) C\left(W_{1}\right)$ y tipos semicálidos sub-húmedos con lluvias en verano, con una cantidad de lluvia invernal entre 5 y $102 \mathrm{~mm}$, con precipitación media anual de $8001200 \mathrm{~mm}$ y una temperatura media anual de $12-16^{\circ} \mathrm{C}$. La geología de la región consiste en rocas ígneas extrusivas ácidas y basaltos del cretásico superior e inferior. Las rocas son del tipo sedimentario y vulcanoso sedimentario. Los suelos encontrados son Litosoles, Cambisoles, Regosoles, y Feozems.

Por su ubicación geográfica, la zona presenta diversas condiciones de vegetación que va desde selva baja caducifolia, masas puras de encino y pino y bosque mezclados de pino-encino. Las especies de mayor valor comercial, tanto por las características tecnológicas de su madera como por su rango de distribución son las del género Pinus. Las especies listadas en orden de importancia por su volumen de aprovechamiento son: $P$. cooperi Blanco, $P$. durangensis Martínez, $P$. leiophylla Schl et Cham, $P$. engelmannii Carr, $P$. cooperi var ornelasi, $P$. Teocote et Cham, $P$. herrerae Martínez. Otras especies de menor valor comercial, distribución y abundancia son $P$. ayacahuite Ehrenb, $P$. lumholtzii Robe et Fern, $P$. douglasiana Martínez, $P$. michoacana var cornuta Martínez, $P$. oocarpa Schiede. Además se aprovechan algunas especies de encino (Quercus spp) y otras especies asociadas con las coníferas y hojosas de los géneros Arbutus, Juniperus, Pseudotsuga, Abies y Picea.

\section{Metodología.}

La base de datos con las variables diámetro $(D)$, altura $(H)$ y volumen $(V)$ a una edad dada se integró con información de análisis troncales provenientes de árboles ubicados en las diferentes condiciones de sitio del área que comprende la Unidad de Conservación y Desarrollo Forestal No 6. La estimación de alturas derivadas de los análisis se realizó de acuerdo al método ISSA propuesto por Fabbio et al. (1994). El volumen se determinó usando la fórmula de Smalian y la fórmula del cono para el diámetro de la parte distal del fuste.

La muestra incluyó solamente arbolado creciendo libre de competencia e incluyó varias clases de edad. Para ajustar los modelos de crecimiento e incremento la muestra comprendió 42 árboles de $P$. cooperi, 39 de $P$. durangensis, 37 de $P$. engelmannii, 35 de $P$. leiophylla y 29 de $P$. herrerae. Para validar los modelos se usó una muestra independiente de datos con 23 árboles de cada especie.

\section{Ecuaciones utilizadas.}

Las ecuaciones de crecimiento utilizadas fueron aquellas reportadas por Zeide (1993), las cuales se ajustaron a los datos de diámetro-edad, altura-edad y volumen-edad de los árboles muestra. El crecimiento para cada especie fue analizado desde dos niveles 
de aproximación: a) crecimiento en grupo, el cual estima el crecimiento de los árboles a partir de un conjunto de árboles que representan todas las condiciones posibles de calidad de sitio, densidad, edad, tipos de suelos, mezclas de especies, etc., y b) crecimiento individual, el cual estima el crecimiento de cada árbol a través de un factor de ponderación. El ajuste de las ecuaciones de crecimiento para el grupo de árboles fue a través una curva promedio para todos los árboles. En la descripción del crecimiento de árboles individuales, los factores de ponderación utilizados exclusivamente para las variables independientes $D, H$ y $V$ fueron $(H),(D)$ y $\left(D^{2} H\right)$, respectivamente. Es decir, las ecuaciones se corrieron dividiendo $D$ por $H$; $H$ por $D$ y $V$ por $D^{2} H:(D / H)=f(t),(H / D)=f(t)$ y $\left(V / D^{2} H\right)=f(t)$. Este proceso estadístico estima el crecimiento de cada árbol individualmente e integra factores de competencia e índice de sitio. Al respecto Murphy y Shelton (1996) corrigen la varianza del crecimiento en área basal con un parámetro de ponderación que es el área basal promedio de los árboles.

El incremento corriente anual, ICA, del grupo de árboles de las especies estudiadas se observó por medio de la forma diferencial de la curva de crecimiento promedio descrita por los modelos de mejor ajuste para las variables $D, H$ y $V$ respectivamente. La edad a la cual se obtiene el máximo ICA se estimó mediante la segunda derivada parcial identificada por el punto de inflexión. Zeide (1999) menciona que cualquiera de los modelos puede proporcionar este parámetro en el punto de inflexión. La edad a la cual las especies alcanzan el turno técnico fue observada por medio de la intersección de las curvas del ICA e IMA (incremento medio anual).

Las ecuaciones de crecimiento utilizadas fueron:

\begin{tabular}{|c|c|}
\hline 1. Chapman-Richards & $Y=B_{0}\left(1-\exp \left(\left(^{B_{2} t}\right)\right)^{B_{3}}\right.$ \\
\hline 2. Hoss feld IV & $Y=\left(t^{B_{3}}\right) /\left(B_{1}+B_{2} t^{B_{3}}\right)$ \\
\hline 3. Gompertz & $\left.\left.Y=\exp ^{\left.-B_{1}-B_{2} \exp \left(-B^{3 t}\right)\right)}\right)\right)$ \\
\hline 4. Logistic & $Y=B_{0} /\left(1+B_{1}\left(\exp \left(^{-B_{2} t}\right)\right)\right)$ \\
\hline 5. Monom olecul ar & $\left.Y=B_{0}\left(1-\exp \left({ }^{B_{1}-B_{2} t}\right)\right)\right)$ \\
\hline 6. Bertalanffy & $Y=B_{0}\left(1-\exp \left({ }^{B_{1}-B_{2} t}\right)\right)^{B_{3}}$ \\
\hline 7. Korf & $Y=\exp \left(^{B_{0}-\frac{\left(B_{1}\right)}{t^{B_{2}}}}\right)$ \\
\hline 8. Weibull & $Y=B_{0}\left(1-\exp \left(^{-B_{1}\left(t^{B_{2}}\right)}\right)\right)$ \\
\hline 9. Sloboda & $Y=\exp ^{\left(-B_{0}-B_{1} \exp ^{-B_{2} B_{3}}\right)}$ \\
\hline 10. Levakovic III & $Y=B_{0}\left(t^{2} /\left(B_{1}+t^{2}\right)\right)^{B_{2}}$ \\
\hline 11. Schumacher & $Y=B_{0} \operatorname{Exp}\left(^{-B_{1} / t}\right)$ \\
\hline
\end{tabular}

Donde: $Y=$ Variable dasométrica del árbol, $D(\mathrm{~cm}), H(\mathrm{~m}), V\left(\mathrm{~m}^{3}\right) ; t=$ edad (años); $B_{0} \ldots B_{3}=$ parámetros estadísticos. 
El ajuste de los modelos se realizó mediante el procedimiento NLIN ( $\sin$ derivadas parciales, DUD) y REG del paquete SAS para los modelos no-lineales y lineales respectivamente (SAS/STAT Institute Inc., 1987). Los modelos de Schumacher y Logístico fueron linearizados y sus parámetros estimados a partir del procedimiento REG. La validación de los modelos parcialmente resuelve los cambios en la estructura lineal y no lineal en la estimación de parámetros y en el número de coeficientes de cada ecuación. Los modelos de Schumacher y Bertalanffy solo tienen dos parámetros en contraste con el resto de las ecuaciones.

Procedimiento estadístico. Los parámetros de las ecuaciones de crecimiento e incremento se ajustaron por especie con los siguientes datos: Pinus cooperi (566), P durangensis (522), P. engelmannii (506), P. leiophylla (478) y P. herrerae (396). Para las ecuaciones que necesitaron transformaciones, los parámetros estadísticos se calcularon con las transformaciones necesarias, pero los estadísticos: coeficiente de determinación $\left(r^{2}\right)$, el error estándar estimado (EES) y sesgo promedio (SP) se estimaron regresando las variables a sus dimensiones originales.

Las ecuaciones se validaron ajustándolas a una fuente independiente de datos seleccionada aleatoriamente. Los estadísticos de comparación fueron: $r^{2}$, EES y SP para cada una de las variables de cada una de las especies estudiadas. Se espera que los estadísticos de ajuste se aproximen a los estadísticos de validación para concluir la aplicación de la ecuación a cualquier otra fuente aleatoria de datos. Estos estadísticos se estimaron como sigue:

donde: $Y_{i}$ es el valor observado o la variable dependiente, es el promedio de las de los datos observados, , es el valor estimado de la variable dependiente con el modelo y es el número de parámetros de cada modelo.

Los errores de las ecuaciones de crecimiento se analizaron para observar su distribución, aleatoriedad y varianza común y fue requisito en la selección de la mejor

$$
\begin{aligned}
& r^{2}=1-\frac{\sum_{i=1}^{n}(Y i-\hat{Y} i)^{2}}{\sum_{i=1}^{n}(Y i-\bar{Y})^{2}} \\
& E E S=\left[\frac{\sum_{i=1}^{n}\left(Y_{i}-\hat{Y}_{i}\right)^{2}}{n-\kappa-1}\right]^{\frac{1}{2}} \\
& S P=\frac{\sum_{i=1}^{n}\left(Y_{i}-\hat{Y}_{i}\right)}{n}
\end{aligned}
$$

ecuación. Los parámetros kurtosis, sesgo y la prueba de normalidad del los errores se estimaron usando el procedimiento UNIVARIATE en SAS (SAS/STAT Institute Inc., 1987). La mejor ecuación de crecimiento para cada variable analizada de cada especie se determinó de la ponderación de los diferentes parámetros estadísticos, los cuales se calificaron por orden de importancia multiplicando un factor como sigue: $r^{2},\left(x_{7}\right)$ EES $\left(x_{6}\right)$, SP $\left(x_{5}\right), \mathrm{CV}\left(x_{4}\right)$, kurtosis (3), sesgo (2) y $\mathrm{P}>\mathrm{W}\left(x_{1}\right)$. La suma de estos resultados arrojo un registro el cual fue contrastado para la selección de la mejor ecuación. La suma adquirió valores positivos para el $r^{2}$ y la $\mathrm{P}>\mathrm{W}$ y negativos para los demás estadísticos.

\section{Características de los árboles} muestra.

Los estadísticos de las bases de datos se resumen en la tabla 1 y el mapa de distribución de la muestra por categoría de diámetro y altura se presenta en la tabla 2.

\section{RESULTADOS Y DISCUSIONES}

En general, no todas las ecuaciones ajustadas convergieron en los parámetros correctos. Para el diámetro, el procedimiento de ajuste no converge en la solución apropiada para las ecuaciones de Korf (7), Sloboda (9) y Levakovic III (10). Los estadísticos promedio de ajuste y validación de las 8 ecuaciones de crecimiento ajustadas mostraron diferencias notorias, aunque no se 
Tabla 1. Estadísticos de parámetros dasométricos de cinco pinaceas de Durango México, para el ajuste y validación de ecuaciones de crecimiento e incremento.

\begin{tabular}{|c|c|c|c|c|c|c|c|}
\hline \multirow[b]{2}{*}{ Especie } & \multirow[b]{2}{*}{ Estadístico } & \multicolumn{3}{|c|}{ Ajuste } & \multicolumn{3}{|c|}{ Validación } \\
\hline & & $\begin{array}{c}D \\
(\mathrm{~cm})\end{array}$ & $\begin{array}{l}\mathrm{H} \\
(\mathrm{m})\end{array}$ & $\begin{array}{l}\text { Edad } \\
\text { (Años) }\end{array}$ & $\begin{array}{c}D \\
(\mathrm{~cm})\end{array}$ & $\begin{array}{c}\mathrm{H} \\
(\mathrm{m})\end{array}$ & $\begin{array}{l}\text { Edad } \\
\text { (Años) }\end{array}$ \\
\hline \multirow[t]{2}{*}{ P. cooperi } & $\bar{x}$ & 11,9 & 13,4 & 54,9 & 12,4 & 13,2 & 51,1 \\
\hline & $D S$ & 5,9 & 6,8 & 32,7 & 6,4 & 6,9 & 28,3 \\
\hline \multirow[t]{2}{*}{ P. durangensis } & $\bar{x}$ & 10,7 & 12,4 & 50,0 & 11,4 & 12,5 & 54,0 \\
\hline & $D S$ & 5,5 & 6,8 & 289 & 6,2 & 6,7 & 35,9 \\
\hline \multirow[t]{2}{*}{ p. engelmannii } & $\bar{x}$ & 9,3 & 10,6 & 47,3 & 11,4 & 12,5 & 46,9 \\
\hline & $D S$ & 5,3 & 5,5 & 26,3 & 6,0 & 7,1 & 28,6 \\
\hline \multirow[t]{2}{*}{ P. leiophylla } & $\bar{x}$ & 10,4 & 11,9 & 53,6 & 10,4 & 11,9 & 53,6 \\
\hline & $D S$ & 5,9 & 6,5 & 32,5 & 5,9 & 6,5 & 32,5 \\
\hline \multirow[t]{2}{*}{ P. herrerae } & $\bar{x}$ & 9,8 & 11,4 & 50,7 & 10,6 & 11,7 & 53,3 \\
\hline & $D S$ & 5,1 & 5,9 & 29,2 & 5,6 & 5,9 & 31,2 \\
\hline
\end{tabular}

$\bar{x}$ = Promedio; $D S=$ Desviación estándar

realizó ninguna prueba estadística, para las cinco especies estudiadas.

Para el diámetro, la ecuación de Chapman Richards y Weibull mostraron los mas altos registros de ajuste y vallidación (el mayor coeficiente de determinación, menor error estándar y una menor probabilidad de desviación de los errores de la normalidad) (Tabla 3), respectivamente. La ecuación Bertalanffy presentó los registros menores para el ajuste y la validación (el menor coeficiente de determinación, el mayor error estándar, un valor de kurtosis y de sesgo bajo).

Para la altura, cuatro ecuaciones no convergen en parámetros (Hossfeld IV, Korf, Sloboda y Levakovic III). La ecuación de Chapman-Richards registró de nuevo los mas altos valores de ponderación de estadísticos para el ajuste y validación de parámetros (tuvo el mayor coeficiente de determinación, menor coeficiente variación y kurtosis) (Tabla 4). Las ecuaciones Monomolecular y de Gompertz presentaron los menores registros para el proceso de ajuste y validación, respectivamente. En particular, estos modelos tuvieron el menor coeficiente de determinación, el mayor error estándar y sesgo (Tabla 4).

Para el volumen sólo siete ecuaciones convergieron en la solución de parámetros. De nuevo, la ecuación de Chapman-Richards mostró los mejores registros para el ajuste y validación de parámetros. En específico, este modelo tuvo los mejores coeficientes de determinación y variación, los menores sesgos y kurtosis y la mayor probabilidad de que los errores sean normales (Tabla 5). Las ecuaciones Monomolecular y Korf presentaron los menores coeficientes de determinación y los errores estándares más altos. 
Tabla 2. Mapa de distribución de la muestra para ajustar y validar ecuaciones de crecimiento e incremento de cinco pináceas de El Salto, Durango, México.

\begin{tabular}{|c|c|c|c|c|c|c|c|c|}
\hline \multirow{2}{*}{$\begin{array}{c}\text { Categoría. } \\
\text { de altura } \\
(\mathrm{m})\end{array}$} & \multicolumn{8}{|c|}{$\begin{array}{l}\text { Categoría de diámetro } \\
(\mathrm{cm})\end{array}$} \\
\hline & $E_{i}$ & 5 & 10 & 15 & 20 & 25 & $>=30$ & Total \\
\hline \multirow[t]{5}{*}{5} & 1 & 1(1) & & & & & & 1(1) \\
\hline & 2 & (1) & 1 & & & & & 1(1) \\
\hline & 3 & 1(1) & & & & & & 1(1) \\
\hline & 4 & 1(1) & 1(1) & & & & & $2(2)$ \\
\hline & 5 & 1(1) & & & & & & 1(1) \\
\hline \multirow[t]{5}{*}{10} & 1 & 1(1) & & & & & & 1(1) \\
\hline & 2 & & 1(1) & 1(1) & & & & $2(2)$ \\
\hline & 3 & 1 & $3(1)$ & 1(2) & & & & $5(3)$ \\
\hline & 4 & & $1(2)$ & 1 & & & & 2(2) \\
\hline & 5 & & $5(1)$ & 1(1) & & & & $6(2)$ \\
\hline \multirow[t]{5}{*}{15} & 1 & & (2) & $6(3)$ & $5(3)$ & & & $11(8)$ \\
\hline & 2 & & $2(3)$ & $6(2)$ & 1(1) & & & $9(6)$ \\
\hline & 3 & & $8(1)$ & $6(4)$ & 1(1) & & & $15(6)$ \\
\hline & 4 & & $2(2)$ & $5(3)$ & $5(1)$ & & & $12(6)$ \\
\hline & 5 & & $1(2)$ & $2(2)$ & $3(1)$ & & & $6(5)$ \\
\hline \multirow[t]{5}{*}{20} & 1 & & & $2(2)$ & $9(2)$ & 1(1) & & $12(5)$ \\
\hline & 2 & & 2 & $8(3)$ & 1(3) & $2(1)$ & & $13(7)$ \\
\hline & 3 & & 1 & $2(2)$ & 1(2) & 1(1) & 1 & $6(5)$ \\
\hline & 4 & & & $3(2)$ & $5(3)$ & 1(1) & 1 & $10(6)$ \\
\hline & 5 & & & $5(4)$ & $4(3)$ & (1) & & $9(8)$ \\
\hline \multirow[t]{5}{*}{25} & 1 & & & & $10(2)$ & $3(3)$ & 1(1) & $14(6)$ \\
\hline & 2 & & & & $3(1)$ & $3(2)$ & (1) & $6(4)$ \\
\hline & 3 & & & & $3(3)$ & 1(1) & 1(1) & $5(5)$ \\
\hline & 4 & & & 2 & $2(1)$ & $2(2)$ & 1(1) & $7(4)$ \\
\hline & 5 & & & & $4(2)$ & $1(2)$ & 1(1) & $6(5)$ \\
\hline \multirow[t]{5}{*}{$>=30$} & 1 & & & 1 & 1 & 1(1) & (1) & $3(2)$ \\
\hline & 2 & & & & 1 & $2(1)$ & $5(2)$ & $8(3)$ \\
\hline & 3 & & & & 1(1) & 1(1) & $3(1)$ & $5(3)$ \\
\hline & 4 & & & & & 1(1) & $1(2)$ & $2(3)$ \\
\hline & 5 & & & & & (1) & $1(1)$ & $1(2)$ \\
\hline Total & & $\begin{array}{c}6 \\
(6)\end{array}$ & $\begin{array}{c}28 \\
(16)\end{array}$ & $\begin{array}{c}52 \\
(31)\end{array}$ & $\begin{array}{c}60 \\
(30)\end{array}$ & $\begin{array}{c}20 \\
(20)\end{array}$ & $\begin{array}{c}16 \\
(12)\end{array}$ & $\begin{array}{c}182 \\
(115)\end{array}$ \\
\hline
\end{tabular}

NOTA: $\mathrm{E}=\mathrm{Especie}(\mathrm{i}=1=P$. cooperi, $2=P$. durangensis, $3=P$. engelmannii, $4=P$. leiophylla y $5=P$. herrerae); $1(1)=$ Número de árboles para el ajuste(Número de árboles para validar). 
Tabla 3. Estadísticos promedio del ajuste y validación de ecuaciones de crecimiento en diámetro para el grupo de árboles de cinco especies de pino en Durango, México.

\begin{tabular}{|c|c|c|c|c|c|c|c|}
\hline \multirow{3}{*}{ Ecuación ${ }^{a}$} & \multicolumn{4}{|c|}{ Estadísticos de la ecuación } & \multicolumn{3}{|c|}{ Estadísticos de los errores } \\
\hline & $\mathrm{R}^{2}(\%)$ & SEE & SP & C.V & Kurtosis & Sesgo & $\operatorname{Pr}<W$ \\
\hline & \multicolumn{3}{|c|}{ AJUSTE } & & & & \\
\hline Chapman-Richards & 68 & 3,14 & 0,01 & 30,3 & 0,55 & 0,37 & 0,000 \\
\hline Hossfield IV & 68 & 3,14 & $-0,03$ & 30,3 & 0,54 & 0,38 & 0,001 \\
\hline Gompertz & 67 & 3,17 & 0,02 & 30,7 & 0,44 & 0,39 & 0,000 \\
\hline Logistic & 64 & 3,32 & $-0,13$ & 32,0 & 0,49 & 0,48 & 0,001 \\
\hline Monomolecular & 67 & 3,19 & $-0,03$ & 30,8 & 0,51 & 0,41 & 0,001 \\
\hline Bertalanffy & 63 & 3,35 & 0,26 & 32,4 & 0,39 & 0,11 & 0,001 \\
\hline Weibull & 68 & 3,14 & $-0,03$ & 30,3 & 0,56 & 0,39 & 0,005 \\
\hline \multirow[t]{2}{*}{ Schumacher } & 65 & 3,24 & 0,12 & 31,3 & 0,37 & 0,19 & 0,000 \\
\hline & \multicolumn{3}{|c|}{ VALIDACIÓN } & & & & \\
\hline Chapman-Richards & 70 & 3,29 & 0,79 & 29,3 & 0,19 & 0,37 & 0,018 \\
\hline Hossfield IV & 66 & 3,25 & 0,16 & 31,0 & 0,42 & 0,33 & 0,020 \\
\hline Gompertz & 66 & 3,24 & 0,21 & 30,8 & 0,40 & 0,30 & 0,019 \\
\hline Logistic & 67 & 3,50 & 0,70 & 31,1 & 0,11 & 0,54 & 0,019 \\
\hline Monomolecular & 70 & 3,28 & 0,76 & 29,2 & 0,21 & 0,36 & 0,019 \\
\hline Bertalanffy & 65 & 3,58 & 1,11 & 31,9 & 0,26 & 0,27 & 0,020 \\
\hline Weibull & 71 & 3,28 & 0,74 & 29,2 & 0,19 & 0,38 & 0,018 \\
\hline Schumacher & 68 & 3,41 & 0,95 & 30,4 & 0,06 & 0,26 & 0,015 \\
\hline
\end{tabular}

$\mathrm{a}=$ Ecuaciones que convergieron en el ajuste de parámetros; $\mathrm{C} . \mathrm{V}=$ Coeficiente de variación.

Las ecuaciones de crecimiento para árboles individuales que registraron valores mayores fueron el Monomolecular y Weibull para el ajuste y validación del crecimiento en diámetro, respectivamente, aunque para el ajuste cuatro modelos mostraron registros similarmente altos. Para la validación, el modelo Weibull sí presenta el mejor registro y en específico muestra el menor EES, CV y los errores menos anormales (Tabla 6).
Para la altura y el volumen, las ecuaciones de Chapman-Richards y Gompertz tuvieron también los mejores registros. Los coeficientes de determinación y sesgo fueron los mejores para el ajuste en la ecuación de Chapman-Richards y el sesgo, kurtosis y sesgo promedio en la ecuación de Gompertz en la validación (Tabla 7 y 8).

Para el volumen, las ecuaciones Bertalanffy y Schumacher presentaron los menores ajustes. 
Tabla 4. Estadísticos promedio del ajuste y validación de ecuaciones de crecimiento en altura para el grupo de árboles de cinco especies de pino en Durango, México.

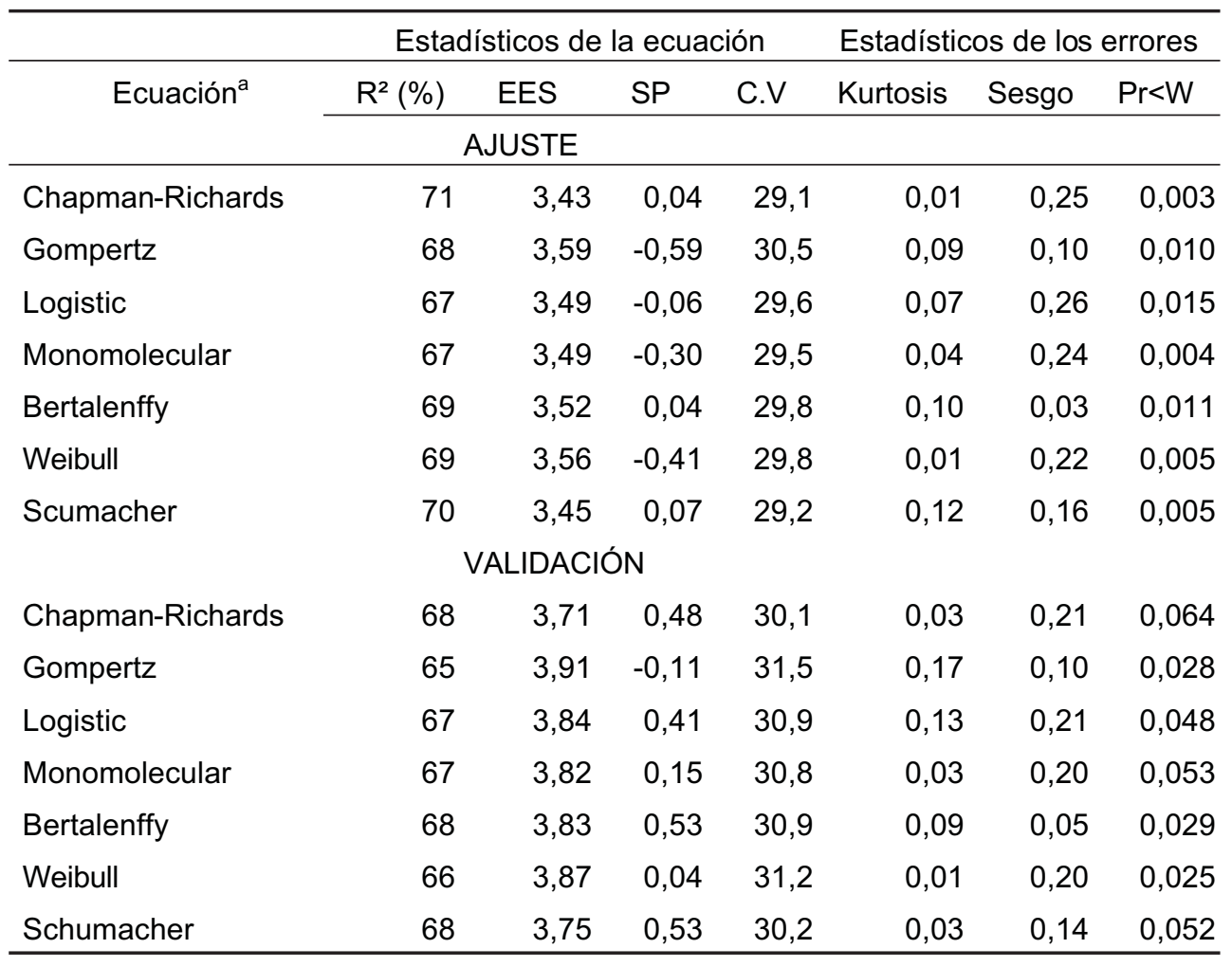

$a=$ Ecuaciones que convergieron en el ajuste de parámetros; C.V = Coeficiente de variación.

La comparación de los dos tipos de ecuaciones, para el grupo de árboles y para cada uno de los árboles resulta en que el EES se reduce a $77 \%$ en la predicción del diámetro, a $12 \%$ en la predicción de la altura y a $17 \%$ en la predicción del volumen. Es decir, las ecuaciones para la proyección de los parámetros dasométricos incrementan su eficiencia cuando se ponderan para predecir el crecimiento individual de los árboles que componen la masa forestal. Una desventaja de este tipo de modelos es la utilización de las dimensiones especificas del arbolado a cada instante en tiempo para su proyección inmediata futura.

La ecuación de Chapman-Richards consistentemente presenta los registros mas altos en la proyección del diámetro, altura y volumen, preferencialmente para el ajuste y en menor medida para la validación para las ecuaciones para el grupo de árboles y para las ecuaciones para árboles individuales. Con el uso de la ecuación recomendada, los coeficientes de variación para estimar el crecimiento en altura y diámetro a nivel grupo de árboles oscilaron de 29 a $30 \%$, mientras que a nivel de árboles individuales para estas variables fue de 22 a $23 \%$ respectivamente. Para estimar el crecimiento en volumen con la misma ecuación, los coeficientes de variación promedio para el grupo de árboles fueron de 82 a $85 \%$, mientras que a nivel árbol individual disminuyeron notablemente entre 19 y $20 \%$. 
Tabla 5. Estadísticos promedio del ajuste y validación de ecuaciones de crecimiento en volumen para el grupos de árboles de cinco especies de pino en Durango, México.

\begin{tabular}{|c|c|c|c|c|c|c|c|}
\hline \multirow{3}{*}{ Ecuación ${ }^{a}$} & \multicolumn{4}{|c|}{ Estadísticos de la ecuación } & \multicolumn{3}{|c|}{ Estadísticos de los errores } \\
\hline & $\mathrm{R}^{2}(\%)$ & EES & SP & C.V & Kurtosis & Sesgo & $\mathrm{Pr}<\mathrm{W}$ \\
\hline & \multicolumn{3}{|c|}{ AJUSTE } & & & & \\
\hline Chapman-Richards & 57 & 0,17 & $-0,00$ & 84,9 & 4,1 & 1,3 & 0,027 \\
\hline Gompertz & 56 & 0,17 & $-0,01$ & 84,8 & 4,1 & 1,3 & 0,002 \\
\hline Logistic & 56 & 0,17 & $-0,01$ & 84,7 & 4,5 & 1,4 & 0,027 \\
\hline Monomolecular & 46 & 0,19 & $-0,04$ & 94,5 & 4,3 & 1,4 & 0,024 \\
\hline Bertalanffy & 56 & 0,17 & 0,01 & 85,0 & 4,0 & 1,3 & 0,000 \\
\hline Korf & 41 & 0,20 & $-0,05$ & 98,0 & 3,7 & 1,3 & 0,000 \\
\hline \multirow[t]{2}{*}{ Schumacher } & 54 & 0,18 & 0,01 & 86,4 & 3,3 & 1,0 & 0,000 \\
\hline & \multicolumn{3}{|c|}{ VALIDACIÓN } & & & & \\
\hline Chapman-Richards & 57 & 0,20 & 0,02 & 82,3 & 3,9 & 1,4 & 0,002 \\
\hline Gompertz & 56 & 0,20 & 0,02 & 82,8 & 3,9 & 1,5 & 0,000 \\
\hline Logistic & 54 & 0,21 & 0,02 & 85,1 & 4,1 & 1,6 & 0,001 \\
\hline Monomolecular & 45 & 0,22 & $-0,01$ & 89,0 & 3,6 & 1,5 & 0,000 \\
\hline Bertalanffy & 56 & 0,20 & 0,03 & 83,6 & 3,7 & 1,5 & 0,001 \\
\hline Korf & 48 & 0,22 & $-0,02$ & 90,1 & 3,1 & 1,3 & 0,001 \\
\hline Schumacher & 57 & 0,20 & 0,03 & 82,3 & 3,4 & 1,3 & 0,000 \\
\hline
\end{tabular}

a= Ecuaciones que convergieron en el ajuste de parámetros; $C . V=$ Coeficiente de variación

La ecuación seleccionada presenta ventajas en comparación con el resto de los modelos las cuales han sido discutidas por investigadores como Zeide (1993) y Elfving y Kiviste (1997), con características como que nace del origen, representa bien la sigmoidal, posee una asíntota (máximo crecimiento) cuyo valor se aproxima por el parámetro Bo y tiene un punto de inflexión. Los parámetros de la ecuación para el grupo de árboles y árboles individuales, para cada una de las especies, para cada una de las variables dasometricas se presentan en el Tabla 9

La ecuación de Chapman-Richards para el grupo de árboles es recomendable para el calculo de variables dasométricas para masas regulares y uniespecíficas, donde la variación en las dimensiones de los árboles no es tan notoria. Teóricamente este modelo podría ser mas adecuado para plantaciones forestales. Los modelos ponderados son recomendables para aquellos rodales que son bastante irregulares en su estructura y los árboles presentan velocidades diferenciales de crecimiento.

El uso de estos modelos resulta en una aproximación a los niveles de rendimiento de estas especies para el área de estudio, puesto que no están calibrados por la competencia dentro del rodal y por factores del rodal tales como calidad de sitio, mortalidad, reclutamiento, etc. 
Tabla 6. Estadísticos promedio del ajuste y validación de ecuaciones de crecimiento en diámetro para árboles individuales de cinco especies de pino en Durango, México.

\begin{tabular}{|c|c|c|c|c|c|c|c|}
\hline \multirow[b]{2}{*}{ Ecuación $^{a}$} & \multicolumn{4}{|c|}{ Estadísticos de la ecuación } & \multicolumn{3}{|c|}{ Estadísticos de los errores } \\
\hline & $\mathrm{R}^{2}(\%)$ & EES & SP & C.V & Kurtosis & Sesgo & $\mathrm{Pr}<\mathrm{W}$ \\
\hline \multicolumn{8}{|c|}{ AJUSTE } \\
\hline Chapman-Richards & 82 & 2,35 & 0,12 & 22,6 & 2,66 & 0,61 & 0,026 \\
\hline Hossfeld IV & 82 & 2,35 & 0,28 & 22,6 & 2,57 & 0,50 & 0,074 \\
\hline Gompertz & 58 & 3,54 & $-2,07$ & 33,9 & 1,25 & $-0,10$ & 0,191 \\
\hline Logistic & 82 & 2,35 & 0,16 & 22,6 & 2,69 & 0,61 & 0,040 \\
\hline Monomolecular & 82 & 2,34 & 0,12 & 22,5 & 2,64 & 0,62 & 0,027 \\
\hline Bertalanffy & 82 & 2,36 & 0,31 & 22,7 & 2,69 & 0,58 & 0,024 \\
\hline Korf & 82 & 2,35 & 0,07 & 22,6 & 2,53 & 0,40 & 0,000 \\
\hline Weibull & 82 & 2,36 & 0,17 & 22,7 & 2,59 & 0,52 & 0,027 \\
\hline Schumacher & 82 & 2,34 & 0,23 & 22,5 & 2,60 & 0,47 & 0,024 \\
\hline \multicolumn{8}{|c|}{ VALIDACIÓN } \\
\hline Chapman-Richards & 82 & 2,54 & 0,54 & 22,6 & 0,46 & 0,24 & 0,026 \\
\hline Hossfeld IV & 82 & 2,54 & 0,70 & 22,6 & 0,49 & 0,14 & 0,001 \\
\hline Gompertz & 70 & 3,31 & $-1,71$ & 29,5 & 0,01 & $-0,35$ & 0,000 \\
\hline Logistic & 82 & 2,54 & 0,58 & 22,6 & 0,45 & 0,22 & 0,040 \\
\hline Monomolecular & 82 & 2,55 & 0,53 & 22,7 & 0,56 & 0,20 & 0,027 \\
\hline Bertalanffy & 82 & 2,56 & 0,73 & 22,7 & 0,47 & 0,21 & 0,012 \\
\hline Korf & 82 & 2,48 & 0,48 & 22,1 & 0,42 & 0,09 & 0,001 \\
\hline Weibull & 82 & 2,42 & 0,50 & 22,1 & 0,45 & 0,14 & 0,030 \\
\hline Schumacher & 82 & 2,53 & 0,65 & 22,5 & 0,52 & 0,11 & 0,000 \\
\hline
\end{tabular}

$a=$ Ecuaciones que convergieron en el ajuste de parámetros; C.V = Coeficiente de variación.

Del análisis del incremento corriente anual, ICA, para el grupo de árboles por medio de la primera derivada de las ecuaciones de crecimiento de ChapmanRichards para el diámetro, altura y volumen se observaron diferencias notorias entre las especies estudiadas. EI ICA máximo alcanzado en diámetro, fue 0,$31 ; 0,28 ; 0,30$; 0,27 y $0,22 \mathrm{~cm}$ año ${ }^{-1}$ para las especies Pinus cooperi, $P$. durangensis, $P$. engelmannii, $P$. herrerae y $P$. leiophylla, respectivamente. Este se alcanzó a las edades de 16, 16, 5, 8 y 1 años, respectivamente.

Las especies Pinus engelmannii, $P$. herrerae $y$. leiophylla presentan un incremento diamétrico máximo a edades muy jóvenes en contraste con las especies de Pinus cooperi y $P$. durangensis. Los turnos técnicos oscilan de los 30 años para $P$. cooperi y $P$. durangensis hasta los 15,10 y 5 
Tabla 7. Estadísticos promedio del ajuste y validación de ecuaciones de crecimiento en altura para árboles individuales de cinco especies de pino en Durango, México.

\begin{tabular}{|c|c|c|c|c|c|c|c|}
\hline \multirow[b]{2}{*}{ Ecuación ${ }^{a}$} & \multicolumn{4}{|c|}{ Estadísticos de la ecuación } & \multicolumn{3}{|c|}{ Estadísticos de los errores } \\
\hline & $\mathrm{R}^{2}(\%)$ & SEE & Sesgo & C.V & Kurtosis & Sesgo & $\mathrm{Pr}<\mathrm{W}$ \\
\hline \multicolumn{8}{|c|}{ AJUSTE } \\
\hline Chapman-Richards & 83 & 2,65 & 0,30 & 22,5 & 2,62 & $-0,52$ & 0,003 \\
\hline Hossfeld IV & 82 & 2,65 & 0,35 & 22,5 & 2,52 & $-0,49$ & 0,007 \\
\hline Gompertz & 82 & 2,70 & 0,47 & 22,9 & 2,34 & $-0,33$ & 0,118 \\
\hline Logistic & 82 & 2,65 & 0,36 & 22,5 & 2,53 & $-0,50$ & 0,003 \\
\hline Monomolecular & 82 & 2,65 & 0,37 & 22,5 & 2,62 & $-0,51$ & 0,003 \\
\hline Bertalanffy & 82 & 2,65 & 0,38 & 22,5 & 2,66 & $-0,55$ & 0,003 \\
\hline Korf & 82 & 2,68 & 0,47 & 22,7 & 2,57 & $-0,55$ & 0,026 \\
\hline Weibull & 82 & 2,67 & 0,47 & 22,7 & 2,49 & $-0,45$ & 0,081 \\
\hline Sloboda & 82 & 2,67 & 0,40 & 22,7 & 2,56 & $-0,45$ & 0,036 \\
\hline Levakovic III & 82 & 2,68 & 0,51 & 22,8 & 2,40 & $-0,38$ & 0,095 \\
\hline Schumacher & 82 & 2,65 & 0,30 & 22,5 & 2,41 & $-0,40$ & 0,062 \\
\hline \multicolumn{8}{|c|}{ VALIDACIÓN } \\
\hline Chapman-Richards & 82 & 2,72 & $-0,09$ & 22,8 & 0,51 & $-0,16$ & 0,002 \\
\hline Hossfeld IV & 81 & 2,84 & $-0,11$ & 22,9 & 0,51 & $-0,15$ & 0,001 \\
\hline Gompertz & 82 & 2,73 & 0,02 & 22,1 & 0,30 & 0,01 & 0,000 \\
\hline Logistic & 81 & 2,83 & $-0,10$ & 22,9 & 0,51 & $-0,15$ & 0,002 \\
\hline Monomolecular & 81 & 2,82 & $-0,09$ & 22,8 & 0,51 & $-0,16$ & 0,002 \\
\hline Bertalanffy & 81 & 2,81 & $-0,08$ & 22,7 & 0,47 & $-0,16$ & 0,003 \\
\hline Korf & 81 & 2,82 & 0,01 & 22,8 & 0,51 & $-0,21$ & 0,025 \\
\hline Weibull & 82 & 2,79 & 0,02 & 22,6 & 0,42 & $-0,12$ & 0,002 \\
\hline Sloboda & 82 & 2,81 & $-0,05$ & 22,7 & 0,54 & $-0,13$ & 0,001 \\
\hline Levakovic III & 82 & 2,76 & 0,07 & 22,3 & 0,32 & $-0,03$ & 0,000 \\
\hline Schumacher & 81 & 2,83 & $-0,16$ & 22,9 & 0,52 & $-0,10$ & 0,000 \\
\hline
\end{tabular}

$\mathrm{a}=$ Ecuaciones que convergieron en el ajuste de parámetros; $\mathrm{C} . \mathrm{V}=$ Coeficiente de variación.

años para $P$. herrerae, $P$. engelmannii y $P$. leiophylla, respectivamente. El crecimiento mayor lo alcanzó $P$. durangensis después de los 60 años (Figura 1).

El ICA máximo en altura fue alcanzado para Pinus durangensis con $0,35 \mathrm{~m}^{\mathrm{año}}{ }^{-1}, P$. cooperi con $0,39 \mathrm{~m}$ año ${ }^{-1}, P$. leiophylla con $0,32 \mathrm{~m}^{2}$ ño ${ }^{-1}, P$. herrerae con $0,43 \mathrm{~m}_{\text {año }} \mathrm{o}^{-1}$ y $P$. engelmannii con $0,40 \mathrm{~m}^{2}$ ño ${ }^{-1}$. Las edades de máximo incremento fueron 24, 21, 21, 19 y 13 para las especies anteriormente descritas, 
Tabla 8. Estadísticos promedio del ajuste y validación de ecuaciones de crecimiento en volumen para árboles individuales de cinco especies de pino en Durango, México.

\begin{tabular}{|c|c|c|c|c|c|c|c|}
\hline \multirow[t]{2}{*}{ Ecuación $^{a}$} & \multicolumn{3}{|c|}{ Estadísticos de la ecuación } & \multicolumn{4}{|c|}{ Estadísticos de los errores } \\
\hline & $\mathrm{R}^{2}(\%)$ & EES & SP & C.V & Kurtosis & Sesgo & $\mathrm{Pr}<\mathrm{W}$ \\
\hline \multicolumn{8}{|c|}{ AJUSTE } \\
\hline Chapman-Richards & 98 & 0,041 & 0,005 & 20 & 22 & $-1,87$ & 0,001 \\
\hline Gompertz & 96 & 0,049 & 0,014 & 23 & 23 & $-1,71$ & 0,000 \\
\hline Logistic & 97 & 0,041 & 0,006 & 20 & 22 & $-1,92$ & 0,000 \\
\hline Monomolecular & 97 & 0,042 & 0,000 & 21 & 24 & $-2,20$ & 0,000 \\
\hline Bertalanffy & 97 & 0,047 & 0,015 & 22 & 22 & $-1,56$ & 0,000 \\
\hline Schumacher & 97 & 0,047 & $-0,005$ & 23 & 20 & $-1,91$ & 0,000 \\
\hline \multicolumn{8}{|c|}{ VALIDACIÓN } \\
\hline Chapman-Richards & 98 & 0,045 & $-0,001$ & 19 & 10 & $-0,99$ & 0,001 \\
\hline Gompertz & 98 & 0,047 & 0,009 & 19 & 6 & $-0,28$ & 0,000 \\
\hline Logistic & 97 & 0,053 & $-0,001$ & 21 & 10 & $-0,97$ & 0,000 \\
\hline Monomolecular & 97 & 0,053 & $-0,002$ & 22 & 11 & $-0,98$ & 0,000 \\
\hline Bertalanffy & 97 & 0,049 & 0,010 & 20 & 7 & 0,15 & 0,000 \\
\hline Schumacher & 96 & 0,058 & $-0,011$ & 24 & 10 & $-1,30$ & 0,002 \\
\hline
\end{tabular}

$a=$ Ecuaciones que convergieron en el ajuste de parámetros; C.V = Coeficiente de variación.

respectivamente. El turno técnico lo alcanzan las especies a los $45,50,25,40$ y 35 años respectivamente. El mayor crecimiento en altura lo muestra $P$. durangensis después de los 75 años (Figura 2).

Los incrementos anuales en volumen mayores fueron observados en Pinus durangensis $\left(0.012 \mathrm{~m}^{3}\right.$ año $\left.{ }^{-1}\right), P$. cooperi $\left(0,0115 \mathrm{~m}^{3}\right.$ año $\left.{ }^{-1}\right), P$. engelmannii $\left(0,011 \mathrm{~m}^{3}\right.$ año $\left.{ }^{-1}\right), P$. leiophylla $\left(0.008 \mathrm{~m}^{3}\right.$ año-1) y $P$. herrerae $\left(0,0075 \mathrm{~m}^{3}\right.$ año-1 $)$ a edades de 59 , $50,44,88$ y 58 años, respectivamente. Los turnos técnicos del crecimiento en volumen fueron alcanzados antes de los 100 años para $P$. engelmannii (70), $P$. cooperi (80), $P$. durangensis (95), y después de este tiempo para $P$. herrerae (105) y $P$. leiophylla $(155$ años). El mayor crecimiento en volumen lo alcanza $P$. durangensis desde los 75 a los 150 años (Figura 3).

Los resultados anteriores demuestran diferencias en los niveles de productividad que alcanzan las especies en la región. La calidad de sitio es el principal factor que determina el nivel de óptimo rendimiento para las especies estudiadas. Al respecto, el crecimiento de estas especies está determinado por la altitud y por lo tanto la calidad de sitio.

De acuerdo con los resultados arrojados por (UCODEFO No. 6. 1997) Programa de Manejo Forestal (PMF) 19972007 para la región de EI Salto, Durango, se estima que el Pinus cooperi, $P$. durangensis, $P$. engelmanni, $P$. leiophylla y $P$. herrerae tienen un promedio de 200, 350, 13, 112 y 150 árboles por hectárea, respectivamente. Si consideramos estas observaciones las 
especies estudiadas pueden alcanzar incrementos anuales en volumen promedio de 2,$4 ; 4,0 ; 1,4 ; 0,9$ y $1,1 \mathrm{~m}^{3} \mathrm{ha}^{-1}$ año- ${ }^{-1}$. Estos incrementos son similares a aquellos registrados por Návar et al. (1998) para rodales mixtos e irregulares dominados por $P$. durangensis y $P$. cooperi del ejido Vencedores de Durango, México. Incrementos similares

Tabla 9. Parámetros estimados de las ecuaciones seleccionadas para estimar el crecimiento en diámetro, altura y volumen de grupos y árboles individuales de cinco especies de la región de El Salto, Durango, México

\begin{tabular}{cccccccccc}
\hline & & \multicolumn{3}{c}{ Grupo de árboles } & \multicolumn{3}{c}{ Árboles individuales } \\
\cline { 3 - 9 } Especie & Variable DS & $\beta_{1}$ & $\beta_{2}$ & $\beta_{3}$ & $\beta_{1}$ & $\beta_{2}$ & $\beta_{3}$ \\
\hline P. cooperi & $D^{r}$ & 3,0 & 20,299 & 0,027 & 1,527 & 0,859 & 0,079 & 0,568 \\
& $H^{r}$ & 3,5 & 21,663 & 0,037 & 2,192 & 1,136 & 0,062 & 0,627 \\
& $V^{r}$ & 0,2 & 0,709 & 0,042 & 8,365 & $8,2^{-05}$ & 0,095 & 2,142 \\
P. durangensis & $D^{r}$ & 2,9 & 34,939 & 0,011 & 1,205 & 0,831 & 0,154 & 0,912 \\
& $H^{r}$ & 3,7 & 30,287 & 0,021 & 1,640 & 1,163 & 0,078 & 0,513 \\
P. engelmanii & $V^{r}$ & 0,2 & 1,018 & 0,029 & 5,599 & $9,9^{-05}$ & 0,099 & 0,883 \\
& $D^{r}$ & 3,4 & 25,865 & 0,015 & 1,073 & 0,874 & 0,977 & 0,974 \\
& $H^{r}$ & 3,3 & 24,730 & 0,027 & 1,419 & 1,085 & 0,201 & 1,862 \\
& $V^{r}$ & 0,2 & 0,545 & 0,053 & 10,293 & $7,7^{-05}$ & 0,071 & 1,512 \\
& $D^{r}$ & 2,8 & 38,659 & 0,006 & 0,999 & 0,865 & 0,155 & 1,008 \\
& $H^{r}$ & 3,2 & 21,198 & 0,029 & 1,834 & 1,112 & 0,160 & 1,430 \\
& $V^{r}$ & 0,1 & 1,491 & 0,012 & 2,826 & $8,1^{-05}$ & 0,171 & 0,525 \\
& $D^{r}$ & 2,6 & 26,269 & 0,013 & 1,111 & 0,847 & 0,100 & 0,403 \\
& $H^{r}$ & 2,9 & 20,077 & 0,046 & 2,425 & 1,159 & 0,114 & 0,817 \\
& $V^{r}$ & 0,1 & 1,002 & 0,016 & 2,555 & $8,4^{-05}$ & 0,088 & 0,922 \\
\hline
\end{tabular}

NOTA: $r$ = parámetros de la ecuación [1]; DS = Desviación estándar de la ecuación ajustada por la curva promedio. 


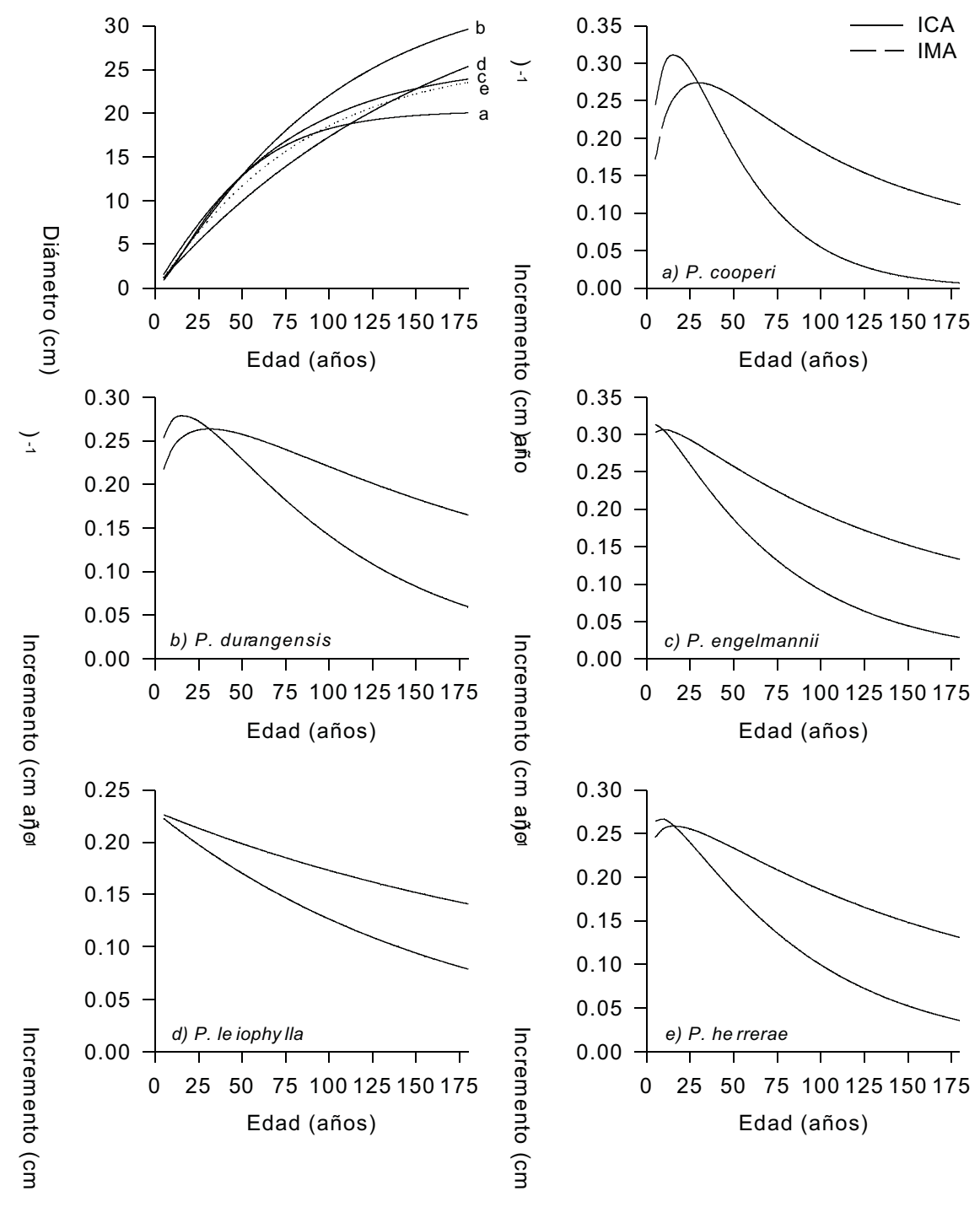

Figura 1. Comparación de las curvas de crecimiento e incremento en diámetro de 5 especies de la región de El Salto, Durango. 

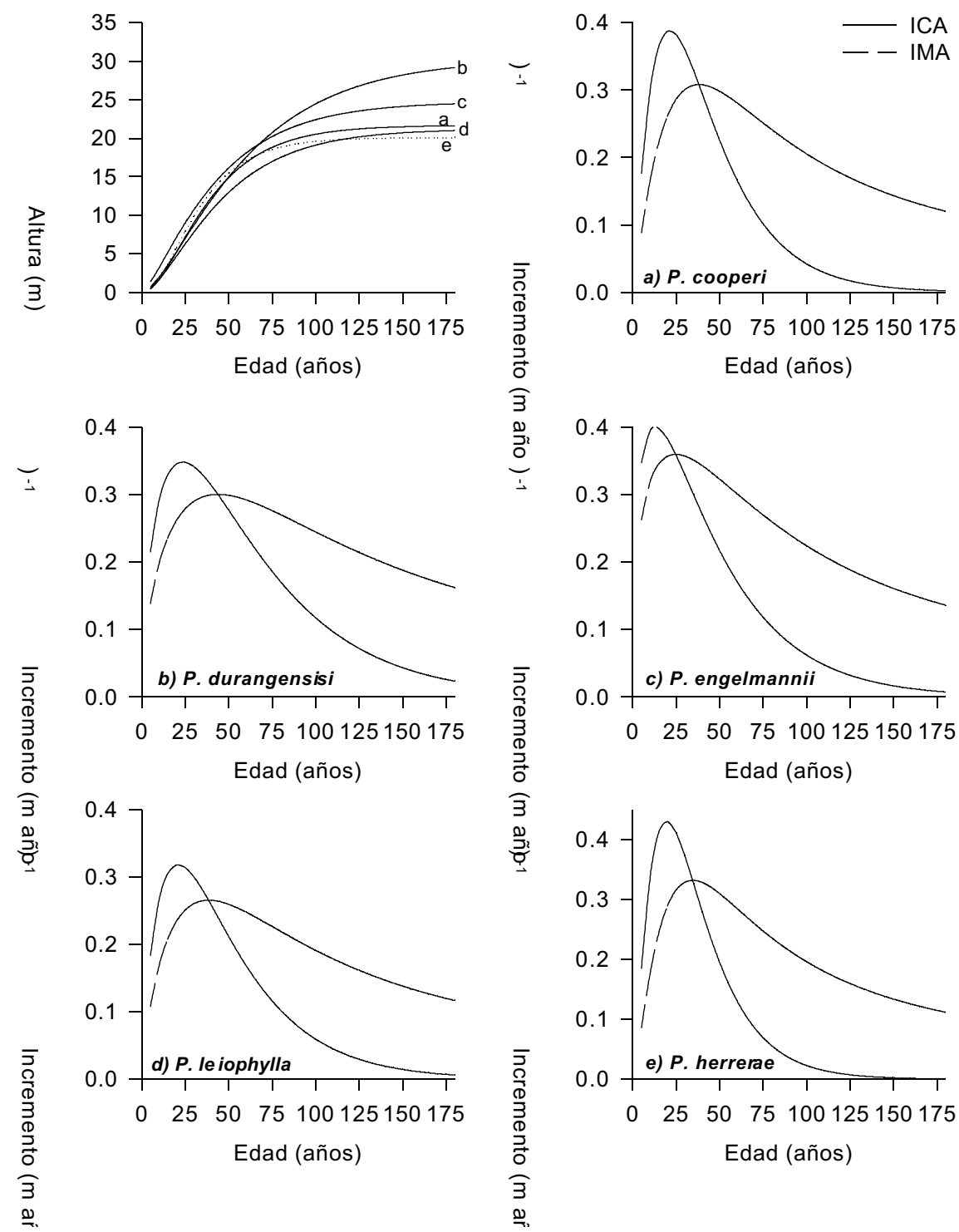

Figura 2. Comparación de las curvas de crecimiento e incremento en altura de 5 especies de la región de El Salto, Durango. 

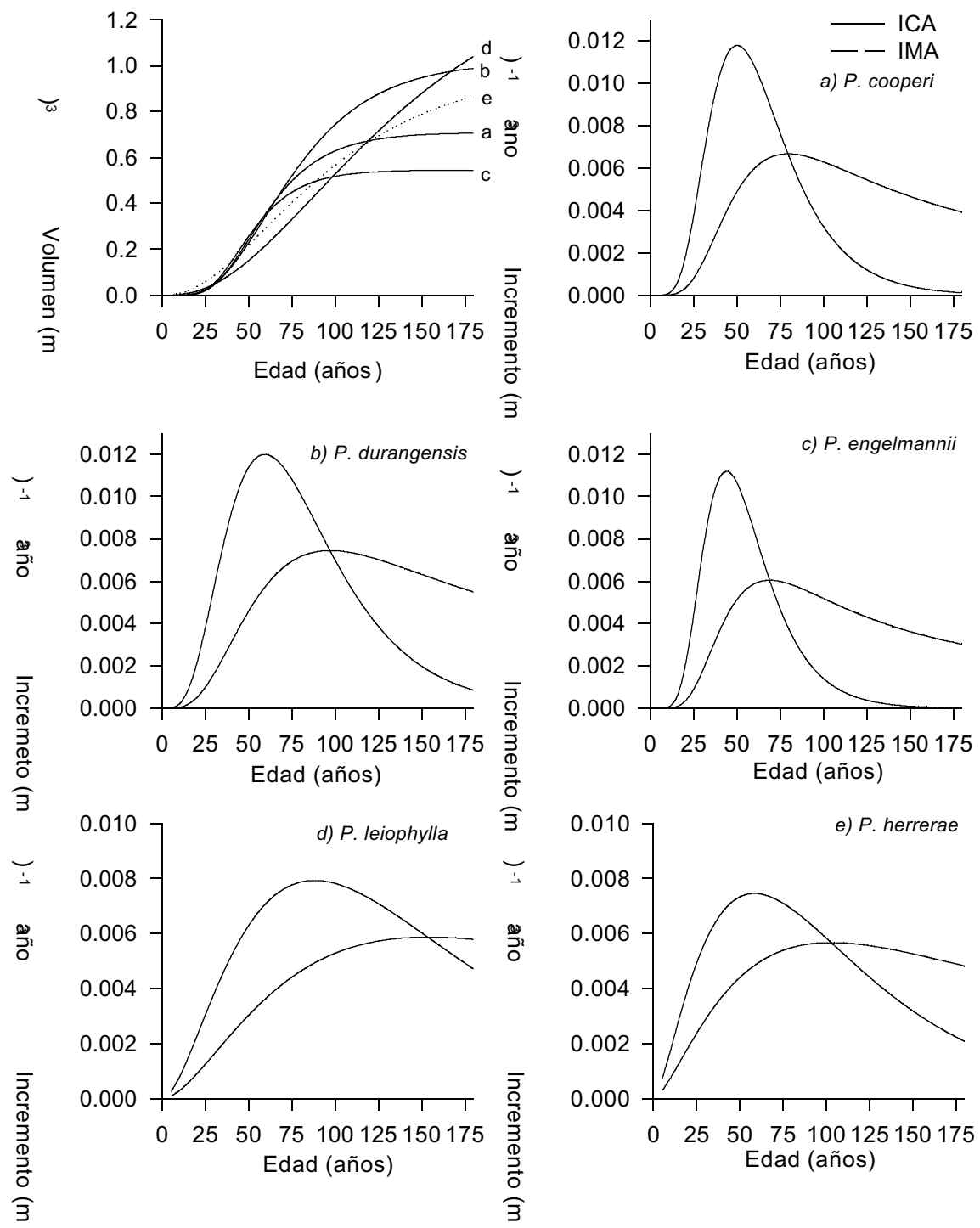

Figura 3. Comparación de las curvas de crecimiento e incremento en volumen de 5 especies de la región de El Salto, Durango. 
fueron determinados en aquellos rodales homogéneos tendientes a la regularidad a partir de datos de análisis de virutas levantados en el inventario forestal de 1996. Para rodales poco productivos (exposiciones sur) y de alta productividad (algunos sitios con suelos profundos de exposiciones norte y zenitales de la parte alta de la Sierra Madre Occidental) el ICA oscila de 0,01 a $8 \mathrm{~m}^{3} \mathrm{ha}^{-1}$ $a n ̃ o^{-1}$, respectivamente. Estos resultados provenientes de la ecuación de ChapmanRichards deben por supuesto validarse adicionalmente con datos provenientes de parcelas permanentes de investigación silvícola para poder ser calibradas adecuadamente. Este es trabajo de investigación adicional.

\section{CONCLUSIONES}

El ajuste y validación de ecuaciones de crecimiento para el grupo de árboles y árboles individuales demostraron que la ecuación de Chapman-Richards resultó modelar consistentemente mejor el crecimiento en diámetro, altura y volumen para las cinco especies estudiadas de la región de El Salto, Durango, México. $P$. durangensis alcanza los crecimientos e incrementos mayores en diámetro, altura y volumen para las condiciones observadas de la región del Salto, Durango.

\section{RECONOCIMIENTOS}

Los autores de este reporte desean hacer patente su agradecimiento al fondo CONAFOR-CONACyT que apoyó este trabajo con financiamiento a través proyecto de investigación 6230 .

\section{REFERENCIAS}

Aguirre, B.C. 1987. Growth and yield models for Pinus cooperi in Durango, México. Ph. D. Dissertation. Colorado State University. Fort Collins, Colorado.

Arney, J.D. 1985. A modeling strategy for the growth projection of managed stands. Can. J. For. Res. 15:1455-1465.
Bahamóndez, V. C. 1995. Modelos de crecimiento individual para renovales de Roble (Nothofagus oblicua (Mirb) (Oerst)) y Rulí (Nothofagus alpina (Poeppet Endl)). Ciencia e Investigación Forestal. 9(1):57-72.

Buckman, R.G y S.R. Shifley. 1983. Guide to evaluating forest growth projection systems. J. For. 81:232-234.

Clutter J.L., J.C. Fortson., J.C. Piennar., L.V. Brister. y R.L. Bailey. 1983. Timber management: A quantitative approach. Wiley. New York. 333 p.

Contreras, J.A., D.L. Otero., M.L. Barrales y H.I. Ojeda. 1996. Estudio de crecimiento de una plantacion de Rauli ubicada en el sector de Panguipulli y expectativas de crecimiento. Bosque 17(1):3-7.

Chojnacky, D.C. 1997. Modeling diameter growth for pinyon and juniper trees in dryland forests. For. Ecol. Manage. 93:21-31.

Elfving, B., y A. Kiviste. 1997. Construction of site index equations for Pinus sylvestris L. using permanent plot data in Sweden. For. Ecol. Manage. 98:125-134.

Fabbio, G., M. Frattegiani y M. Ch. Manetti. 1994. Height estimation in stem analysis using second differences. For. Sci. 40(2):329-340.

Hokka, H. y A. Groot. 1999. An individual-tree basal area growth model for black spruce in second growth peatland stand. Can J. For. Res. 29:621-629.

Huang, S. y S.J. Titus. 1995. An individual tree diameter increment model for white spruce in Alberta. Can. J. For. Res. 25:1455-1465.

Martin, G.L y A.R. Ek. 1984. A comparison of competition measures and growth models for predicting plantation red pine diameter and height growth. For. Sci. 30(3):731-743.

Martínez, M. 1963. Las Pináceas Mexicanas. 


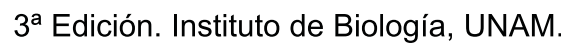
$401 \mathrm{p}$.

Murphy, P.A y M.G. Shelton. 1996. An individual-tree basal area growth model for loblolly pine stands. Can. J. For. Res. 26:327-331.

Návar J., S. Corral y J. Contreras 1998. Perpectivas preliminares para el modelaje del crecimiento de bosques irregulares bajo manejo de pino de Durango, México. Primer Congreso Latinoamericano IUFRO. Validivia, Chile.

Návar, J., J. Jiménez., P.A. Domínguez, O.A. Aguirre, M. Galván, y A. Páez. 1996. Predicción del crecimiento de masas forestales irregulares en base a las distribuciones diamétricas en el sureste de Sinaloa, México. Investigación Agraria. Sis. y Rec. For. 5(2):214-229.

SAS Institute Inc. 1987. SAS/STAT user's guide. Version 6.0 SAS Institute Inc. Cary, N.C.

Shifley, S. R y G.J. Brand. 1984. ChapmanRichards growth function constrained for maximum tree size. For. Sci. 30(4):10661070.

Smith, W.R., R.M. Ferrar Jr., P.A. Murphy., J.L. Yeiser., R.S. Meldahl y J.L. Kush. 1992. Crown and basal area relationships of open-growth southern pines for modeling competition and growth. Can. J. For. Res. 22:341-347.

UCODEFO No. 6. 1997. Memoria general del Programa de Manejo Forestal persistente 1997-2007. Unidad de Conservación y Desarrollo Forestal No. 6. "El Salto", Durango, México.

Vanclay, J.K.. 1995. Growth models for tropical forest: A synthesis of models and methods. For. Sci. 41(1):7-42.

Vanclay, K.V. 1994. Modeling Forest Growth and Yield: Applications to Mixed Tropical Forests. CAB International. Wallingford, Oxford, UK. 312 p.

Wykoff, W.R., N.L. Crookston y A.R. Stage. 1982. User's guide to the stand prognosis model. USDA For. Serv. Gen. Tech. Rep. INT-133.

Zeide, B. 1993. Analysis of growth equations. For. Sci. 42(3):594-616.

Zeide, B. 1999. Pattern of height growth for southern pine species. For. Ecol. Manage. 118:183-196.

Zepeda, B.E.M y A. Domínguez P. 1998. Niveles de incremento y rendimiento maderable de poblaciones naturales de Pinus arizonica Engl., de El Poleo, Chihuahua. Madera y Bosques. 4(1):2739.

Manuscrito recibido el 15 de Abril de 2005.

Aceptado el 20 de Mayo de 2005.

Este documento se debe citar como:

Corral, S. y J. J. Návar C. 2205. Análisis del crecimiento e incremento de cinco pináceas de los bosques de Durango, México. Madera y Bosques 11(1): 29-47. 